\title{
Psychological Analysis of the Extreme Conflict between Teachers and Students
}

\author{
Lili Yang ${ }^{\mathrm{a}}$, Yanhong $\mathrm{Hu}^{\mathrm{b}}$ and Yongfeng $\mathrm{Ma}^{\mathrm{c}}$ \\ Northwest Minzu University, Lanzhou, Gansu, China 730000 \\ a923086575@qq.com, '1107379343@qq.com ,'m15818947078@163.com
}

Keywords: Teacher-Student Conflict, Attack, Psychological Characteristics.

\begin{abstract}
Extreme teacher-student conflict has become a social public hazard, causing more and more attention. This study started from the psychology, by taking the case of scholar-tyrant Yao Shi in Hunan Province as research object, this study analyzes the psychological process and mechanism of extreme teacher-student conflict, and understands the psychological particularity of attackers in terms of cognition, emotion, will and motivation. This study will be helpful for excavate the psychological conflict roots of this special group, and provide psychological basis for further transforming confrontational conflicts into general conflicts, so as to ease the contradictions between teachers and students.
\end{abstract}

\section{Introduction}

The relationship between teachers and students has always been a hot topic in educational research. [1]-[4] Especially the extreme conflict between teachers and students is becoming more and more serious, which has aroused widespread concern in the society. Psychological researchers focus on the analysis of psychological differences between teachers and students. In their view, the teacher-student relationship is a natural interpersonal relationship that maintains a relatively stable and relatively fragile balance. This study attempts to analyze the psychology of teachers and students when extreme teacher-student conflicts occur by analyzing a case of killing a teacher in our country, and get some inspiration from the conflict between teachers and students.

\section{The two manifestations of extreme conflict: aggression and violence}

\subsection{Attack}

According to China's Modern Chinese Dictionary, an attack has two meanings: 1 attack; 2 malicious. In different studies, the definition of an attack has not been unified. In psychology, aggressive behavior is defined as behavior that causes psychological or physical harm to other individuals. In psychiatry, an aggression is an act of harming or destroying an individual other than himself.

\subsection{Violence}

In China's Modern Chinese Dictionary, violence refers to: 1 the power of coercion. 2 refers specifically to the mandatory force of the state. In the fields of psychology and biology, researchers use "attacks" more, and the subjects of behavior include animals and people. In the fields of law, criminology and sociology, researchers use "violence" more and more to describe the behavior of people or state forces.

\section{The psychological process of extreme conflict}

\subsection{Strong desire and choice of satisfaction}

People's needs have different levels of strength and weakness. When it is weak, it is difficult to generate motivation for activity. And the strong desire to taste the motivation of people's behavior. A strong normal desire cannot determine whether a person attacks or does not attack. Only when the desire is very strong, can't wait, and embark on the path of infringing on the interests of others and society to satisfy the desire, will it become the initial link of behavioral attack psychology. 


\subsection{Moral defects and the lack of inhibition.}

The lack of personality of the attacker is due to the formation of social norms in the process of socialization. It is the psychological basis for generating illegal attacks. Compared with a healthy personality, this personality defect has the following characteristics: (1) The desire for demand is strong. (2) Focus on self-interest. (3) An aggressive character. (4) The correct consciousness is weak. (5) Lack of social feelings. Under such circumstances, it is easy to generate intention to attack illegal activities.

\subsection{Intention of attack behavior}

The intention of attack is the intention, impulse or intention of carrying out the attack. It is an undivided and unrecognized need for attack. When the personality of the attacker is obviously defective, or bad habits have been formed, under the stimulation of external incentives, it is possible to produce vague, hazy, no specific direction of the intrinsic impulse to attack, thus forming a stage of germination of attack intention.

\subsection{Form the attack motive and determine the purpose of the attack}

The so-called attack motive is specifically the motivation of the activity that causes the attack behavior. It is the inner cause of the attacker's promotion and attack behavior. Normally, it goes through three stages: attack intention (fuzzy attack intention), behavioral motivation (with clear pointing and aggression target), and attack resolution (from will to decision to will).The purpose of the attack is the result that the attacker hopes to achieve through the attack.

\section{Analysis of the psychological characteristics of a good student who kills a teacher}

In November 2017, Luo, a high school student from Hunan, had a dispute with the class teacher, Mr. Bao, due to academic disputes. He stabbed the teacher with a spring-jumping knife, and the deadly knife was on the teacher's neck. This case shocked the country. By analyzing its particularity, it is helpful to solve the psychological conflict of this special group, which is conducive to transforming the confrontational conflict into a general conflict and alleviating the contradiction between teachers and students.

\subsection{To know characteristics}

\subsubsection{Lack of legal concept}

According to Kohlberg's stage of moral development, Luo is more at a directional stage of customary level and order maintenance. There are two explanations for his behavior: First, Luo learned the law and understood some criminal legal knowledge, but did not form the concept of the rule of law. Second, law-abiding has not yet become an internal need, so it is not possible to use the concept of the rule of law to guide and regulate one's behavior.

\subsubsection{Immature cognitive structure}

According to Piaget's stage of cognitive development, children after the age of 11 and 12 gradually entered the stage of formal computing. When Luo is dealing with conflicts, his thinking is at the level of the pre-operational stage, and the cognitive structure is extremely immature. It is manifested in the following: First, in terms of time, he cannot properly connect things or phenomena with its past and future. stand up. Second, there is a lack of criticism, introspection, self-consciousness, predictability and adaptability. Third, cognition cannot control the influence of emotions and emotions. Once there is a tendency to attack, it becomes difficult to control.

\subsection{The emotional characteristics}

\subsubsection{The primordial and low-level nature of emotions.}

Most of the teenagers pursue physiological needs and sensory stimulation, which is a primitive, low-level need. Emotional changes such as joy, anger, sadness, and joy are often related to the satisfaction of this need. After assassinating the class teacher Bao, Luo said to his daughter, "I killed your father. "This is a kind of emotional expression that is almost abnormal. Perhaps the attacker wants to get the pleasure of venting anger from the scared expression of the victim's daughter. This primitive and bloody emotional demand.

\subsubsection{Extreme instability of emotions}

The specific performances are: emotional changes, impermanence, violent and violent, and happy when moving. If the educators lack feelings for them and adopt a satirical and rude attitude, they will have strong opposition and even the educators will talk back and confront each other. However, Luo's situation is that Bao's special care for him has become a problem for him. 


\subsection{Will features}

\subsubsection{Adventurous}

The attacker's characteristics in the attack are characterized by arrogance, conceit, blindness, and risky bad will. Before the attack, the attacker will have different degrees of fear, but for some purpose, he will cross the heart and take risks. When the attack is going on, the attacker only thinks that he is quick to get out, and he is going out, how is love going. After the discovery, the attacker can get rid of it and get rid of it.

4.3.2 Easily to be implied

Because teenagers distinguish between right and wrong, it is easy to accept the suggestion of others and lead to blind obedience. Luo is a student with excellent academic performance. His tutor is strict. The only source that can be hinted at may be witnessing beatings in the society or TV news and internet reports. That is, the social learning of Bandura. Observed impressions are stored in the brain. After the similar situation is reproduced, the attacker gets psychological hints, which can easily lead to aggressive behavior.

\section{Conclusion}

This paper makes a lot of reference to the field of criminal psychology, trying to dig out the psychological process of juvenile aggression from the criminal psychology, understand the psychological characteristics of the attacker, and solve and resolve the extreme teacher-student conflict at the source. But, because this study does not discuss external factors other than psychological factors, such as family background, personality characteristics, peer relationships, teacher-student relationship, etc., it is not possible to consider the overall situation.

\section{Acknowledgments}

This work was financially supported by the Project "Northwest Minzu Education Research" Innovation Team of "Dominant and Characteristic Discipline" of Northwest Minzu University Supported by "the Fundamental Research Funds for the Central Universities” in 2017 (Project Number: 31920170096)

\section{References}

[1] Yanxia Liu, Teacher-student relationship in different types of knowledge-Three questions on university teacher-student relationship, Higher Education Research, vol.35, pp. 82-86, 2014.

[2] Changwei $\mathrm{Li}$, The change of teacher-student relationship from ancient times to the present, Educational Research, vol.33, pp. 113-119, 2012.

[3] Zuofang Wan, Haibin Ren, Four types of teacher-student relationship: based on the history and practice of education, Educational Theory and Practice, vol. 31, pp. 32-35, 2011.

[4] Xiao Zhang, Xiaoyan Wang, Huichang Chen, Relationship between temperament and teacher-student in early childhood: the role of family emotional environment, Journal of Psychology,vol. 42, pp. 768-778, 2010. 